\title{
Anti-Obesity Therapy by Food Component: Unique Activity of Marine Carotenoid, Fucoxanthin
}

\author{
Kazuo Miyashita* \\ Faculty of Fisheries Sciences, Hokkaido University, Japan
}

\begin{abstract}
Received: November 19, 2013; Accepted: November 29, 2013; Published: December 12, 2013
"Corresponding author: Kazuo Miyashita, Faculty of Fisheries Sciences, Hokkaido University, Japan, Tel: 81-138-408804; Fax: +81 138 408804; E-mail: kmiya@fish.hokudai.ac.jp
\end{abstract}

\begin{abstract}
Obesity represents a rapidly growing threat to the health of populations in an increasing number of countries. Several functional food ingredients are proposed or claimed to benefit weight control through their effects on energy metabolism. A few molecular targets offer the most hope for this kind of anti-obesity therapeutics. One of the keys for success will be induction of uncoupling protein 1 (UCP1). UCP1 is usually expressed in brown adipose tissue (BAT). However, adult humans have very little BAT and most of fat is stored in white adipose tissue (WAT). Considered as breakthrough discoveries for an ideal therapy of obesity, induction of UCP1 expression in tissues other than BAT, especially in WAT, by food constituent would have been expected. From this view point, anti-obesity effect of fucoxanthin should be interested, because it reduces the fat accumulation in abdominal WAT through the UCP1 induction in the WAT. Interestingly, fucoxanthin is effective in subjects with obesity and with metabolic disorders, but not in normal ones. Therefore, fucoxanthin will be a desirable nutraceutical fighting against obesity and related metabolic disorders.
\end{abstract}

Keywords: Obesity; UCP1; WAT; BAT; Fucoxanthin

\section{Introduction}

Obesity is defined as a condition of excess body fat, and it causes or exacerbates many health problems, both independently and in association with other diseases. It also contributes to increased mortality rates by type 2 diabetes mellitus, coronary heart disease (CHD), and cancer. We well know that there are only 2 ways for obesity therapy; they are reduction of energy intake or increase in energy expenditure. Thus, lifestyle interventions, i.e., changed dietary habits and increased physical activity, are basically important for treatment of obesity.

In addition to above essential concept, we should also pay attention to nutritional and dietary factors and metabolically active food compounds that are related to addressing the energy imbalance issue. Major molecular mechanisms for obesity control will be as follows: reduction of food intake through the control of signals from gut and adipose tissue, inhibition of nutrient absorption, increasing thermogenesis to dissipate food energy as heat, and modulation of fat synthesis/lipolysis or adipose differentiation/apoptosis [1]. Many functional food components used for obesity therapy have been shown to alter energy metabolism by influencing fat absorption, substrate utilization rate, and thermo genesis.

Among the effect of these functional components, upregulation of sympathetically mediated thermo genesis should be one of the main molecular targets. Brown adipose tissue (BAT), a major thermogenic tissue, has enormous capacity to produce heat production by adaptive thermo genesis [2,3]. BAT has been found predominantly in hibernating animals, small rodents, and newborns that require active thermo genesis to protect themselves from cold exposure and maintain body temperature. The dissipation of energy is accomplished by the BAT-specific protein, uncoupling protein-1 (UCP1), which generates heat by uncoupling the respiratory chain. UCP1 allows BAT to dissipate the mitochondrial proton electrochemical gradient that is normally used to drive ATP synthesis. Since BAT is also found in healthy adults, showing the possibility to make BAT-mediated dissipation of excess energy even in adult humans (Enerbäck, 2010) [4], a great deal of interest has been focused on the functionality of food components that induced thermo genesis through UCP1 activation.

This review is mainly concerned with the anti-obesity effects of marine Carotenoid, fucoxanthin, as expected food component for anti-obesity therapy.

\section{Fucoxanthin}

Fucoxanthin is a major Carotenoid mainly present in chloroplasts of brown seaweeds. It makes a complex with chlorophyll-protein and plays an important role in light harvesting and photo protection for effective light utilization and up-regulation of photosynthesis [5,6]. Fucoxanthin has a unique structure including an allenic bond and a 5,6-monoepoxide in the molecule (Figure 1) and is found in edible brown seaweeds. In Southeast Asian countries, some brown seaweeds containing fucoxanthin are often used as a food source. Fucoxanthin is obtained as lipid component of brown seaweeds. The brown seaweed lipids usually contain around $5 \%$ weight fucoxanthin per total lipids [7].

Fucoxanthin shows remarkable physiological effects based 


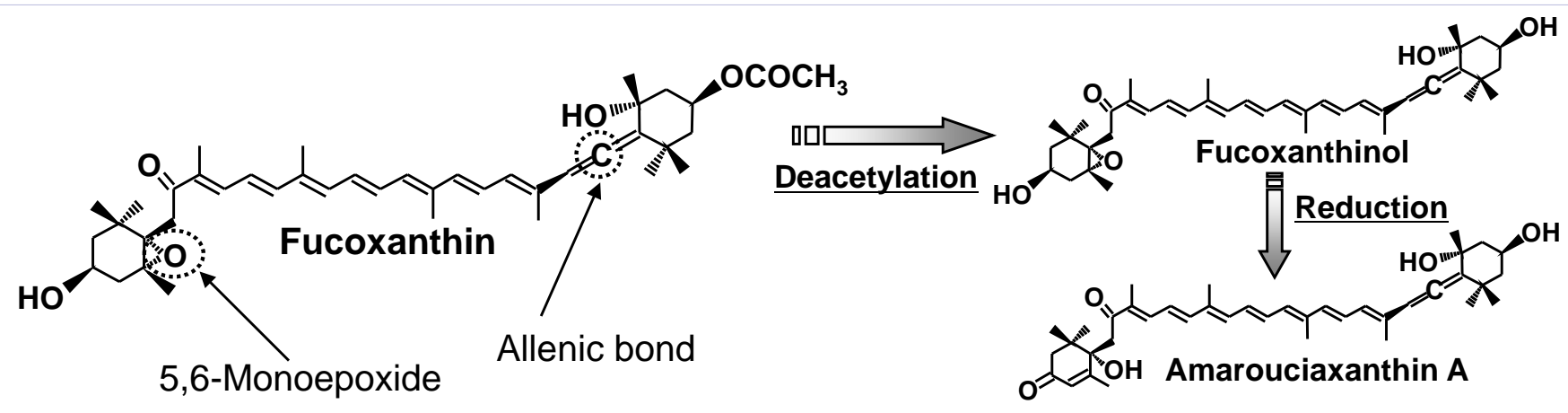

Figure 1: Metabolic Pathway of Fucoxanthin.

on its unique molecular structure as well as general biological activities found in Carotenoid. Carotenoids may decrease the risk of diseases and disorders by reduction of oxidative stress in the target tissues, if they can be present in sufficient amounts and right location to be a meaningful antioxidant in the tissues. For example, lutein and zeaxanthin has been studied widely and proven to show diverse beneficial effects on human health on the basis of their in vivo antioxidant activity, particularly on optimizing eye health [8]. Fucoxanthin has been reported to also show such antioxidant ability by quenching ${ }^{1} \mathrm{O}_{2}$ and scavenging different free radicals [9].

On the other hand, other mechanisms of action that are independent of the antioxidant activity are likely to be more important to understand the biological importance of fucoxanthin. For the better understanding the physiological effect of carotenoids, efforts have been sometimes required to make clear the modulation effect of carotenoids or their metabolites on specific gene and protein expressions in biological systems. The effect is usually based on the interaction between a carotenoid or its metabolite and biological key molecule such as receptor protein and co-activator, where specific chemical structure of carotenoid may be essential for binding to these biomolecules. From this viewpoint, unique mechanism for the anti-obesity effect of brown seaweed carotenoid, fucoxanthin, is interesting, since this effect of fucoxanthin is based on the induction of UCP1 in abdominal white adipose tissue (WAT) mitochondria to lead fatty acid oxidation and heat production in the WAT [10-12].

\section{Anti-obesity effect of fucoxanthin}

Animal experiments clearly showed the anti-obesity effect of fucoxanthin. Significant decrease in body weight and abdominal WAT weight by fucoxanthin intake was found in obese-diabetes model mice $[13,14]$. The decrease of body weight was consistent with that of abdominal WAT weight. On the other hand, BAT weight normalized for body weight increased by fucoxanthin intake. The same effect of fucoxanthin on the body weight and abdominal WAT weight was also observed in normal mice fed high fat diet [15]. In addition, comparative study using both obese model KK- $A^{y}$ mice and lean C57BL/6J mice indicated that fucoxanthin attenuates the excess fat accumulation in abdominal WAT of obese KK- $A^{y}$ mice, while no effect was found in lean C57BL/6J mice fed normal-fat diet [16]. However, feeding of fucoxanthin significantly suppressed abdominal WAT weight of C57BL/6J mice fed high-fat diet to the same level of that found in normal dietary group. These results suggest that suppressive effect of fucoxanthin on the WAT weight gain is specific for adiposity in the development of obesity in mice. This specificity will be important for the safe application of fucoxanthin to human obese therapy.

Significant effect of fucoxanthin on the decrease in abdominal WAT weight of obese model mice appeared atleast more than $60 \mathrm{mg}$ fucoxanthin intake/kg body weight/day [17]. On the other hand, recent study demonstrated the significant reduction of abdominal WAT of obese female volunteers by intake of fucoxanthin less than $0.024 \mathrm{mg} / \mathrm{kg} /$ day $(2.4 \mathrm{mg}$ intake/day for volunteers with $100 \mathrm{~kg}$ average weight) [1]. This difference in the effectiveness between rodents and human may be due to the different absorption rate and/or to different sensitivity to fucoxanthin.

Several food components are currently being promoted for weight management and obesity therapy $[18,19]$. The potential biological mechanisms include increased energy expenditure, increased fat oxidation, decreased fat absorption, and increased satiety through the regulation of related signaling pathways and specific molecular targets mainly in adipose tissue and liver. Major target of fucoxanthin is abdominal WAT, because more than $80 \%$ of fucoxanthin metabolites were accumulated in abdominal WAT, when purified fucoxanthin containing diet (100 mg fucoxanthin/100 g diet) was given to mice. The metabolic pathway of fucoxanthin is well documented [2023]. As soon as after absorption, fucoxanthin is deacetylated to form fucoxanthinol, and then, some of the fucoxanthinol can be reduced to amarouciaxanthin A Figure 1. Dietary fucoxanthin preferentially accumulates as amarouciaxanthin A in the abdominal WAT and as fucoxanthinol in the other tissues; therefore, the main active form of fucoxanthin in abdominal WAT will be amarousiaxanthin A. Furthermore, molecular level analysis showed the UCP1 induction by fucoxanthin as main mechanism for the anti-obesity effect of fucoxanthin.

UCP1 in abdominal WAT as a molecular target for fucoxanthin

When fucoxanthin was fed to mice, UCP1 gene and protein 
expressions were induced in abdominal WAT, showing that the decrease in abdominal WAT weight of fucoxanthin intake in rodents would be due to the up-regulation of thermo genesis through UCP1 expression in abdominal WAT (Figure 2) [13]. It is no doubt that UCP1 is a key molecule for anti-obesity. UCP1 expression is known as a significant component of whole body energy expenditure and its dysfunction contributes to the development of obesity. However, UCP1 is mainly expressed in BAT usually not in WAT. Therefore, anti-obesity effect of fucoxanthin through UCP1 induction in WAT is unique.

The UCP1 allows BAT to dissipate the electrochemical gradient that is normally used to drive adenosine triphosphate synthesis, generating heat by this uncoupling oxidative phsphorylation. Thermogenic activity of BAT is dependent on UCP1 expression level controlled by the sympathetic nervous system via noradrenaline that is stimulated by cold, adrenergic stimulation, $\beta 3$-agonists, retinoids and thyroid hormone [2428]. UCP1 up-regulation or stimulation is dependent on several key bio-molecules such as $\beta 3$-adorenarine receptor $(\beta 3 \mathrm{Ad})$, peroxisome proliferator-activated receptor gamma co-activator 1 (PGC-1), and peroxisome proliferator-activated receptor- $\gamma$ (ППАР $\gamma$ ).

Although UCP1 expression is the signature of BAT, it has been also found in WAT of mice over expressing fork head box protein $\mathrm{C} 2$ (FoxC2), a winged helix gene, with a change in steady-state levels of several WAT and BAT derives mRNAs [29]. This result suggests the possibility of UCP1 expression even in WAT. Although the mechanism for the UCP1 expression in abdominal WAT by fucoxanthin supplementation has not yet been completely made clear, up-regulation of several factors such as $\beta 3 \mathrm{Ad}$ and PGC- 1 would be a key event for the explanation of the fucoxanthin activity. The UCP1-expressing brown-like adipocytes can be recruited in WAT by prolonged cold exposure or by treatment with $\beta$-adrenergic agonists [30]. Fucoxanthin may be related to the induction of this brown-adipose like cell formation in abdominal WAT.

\section{Conclusion}

Several food components are known to increase energy expenditure to improve obesity condition. A number of proposed functional foods have been shown to act to alter energy expenditure or appetite control. Several food components can inhibit fat absorption, influence carbonyl oxidation, or increase energy expenditure, satiety, and fat oxidation [19, 31, 32]. Among them a great deal of interest has been focused on increase in energy expenditure controlled by the sympathetic nervous system via noradrenaline that is stimulated by cold, adrenergic

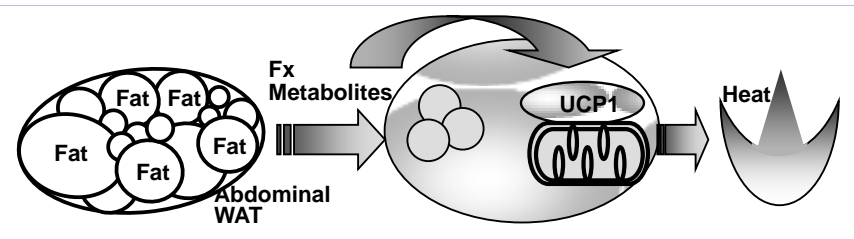

Figure 2: Induction of UCP1 in abdominal WAT by fucoxanthin intake. stimulation, $\beta 3$-agonists, retinoids and thyroid hormone. Increase in noradrenaline secretion by activation of the central nervous system makes it possible to increase the usage of adenosine triphosphate through ion pumping and substrate cycling and to increase the rate of mitochondrial oxidation with poor coupling of ATP synthesis leading to increased heat production. Food ingredients such as capsaicin, a major pungent principle in red hot peppers, have been reported to increase thermogenesis by dose-dependent enhancement of noradrenaline secretion followed by $\beta_{3}$-adrenergic stimulation [33-35] .

Because of its capacity for uncoupled mitochondrial respiration, BAT has implicated as an important site of facultative energy expenditure in small rodents. This has led to speculation that BAT normally functions to prevent obesity. In fucoxanthinfed mice, BAT weight was significantly greater than that in control mice, while abdominal WAT weight decreased by fucoxanthin feeding [13-15]. Interestingly, the fucoxanthin supplementation also induced UCP1 expression even in abdominal WAT Figure 2. These findings show that the decrease in abdominal WAT weight of fucoxanthin intake in rodents is mainly due to the up-regulation of thermogenesis through UCP1 expression both in BAT and abdominal WAT. The UCP1 induction in WAT will be related to the appearance of inducible brown adipcoytes in traditional white fat depots [2]. UCP1-based thermo genesis has been shown to be a powerful thermogenic system for oxidation of excess fat to reduce obesity [36-38]. UCP1 is usually expressed only in BAT; however, BAT content in adult human is scanty. Therefore, the possible ability of fucoxanthin to convert white adipose cell to brown adipose cell may open new anti-obesity therapy by food components.

\section{References}

1. Bary, G. A., \& Tartaglia, L. A. (2000). Medicinal strategies in the treatment of obesity. Nature, 404(6778), 672-677.

2. Kozak, L. P. (2010). Brown fat and the myth of diet-induced thermogenesis. Cell Metab, 11(4), 263-267.

3. Seale, P. (2010). Transcriptional control of brown adipocyte development and thermogenesis. Int J Obes (Lond), 34, S17-S22.

4. Enerbäck, S. (2010). Brown adipose tissue in humans. International Journal of Obesity, 34, S43-S46.

5. Eonseon, J., Polle, J. E. W., Lee, H. K., Hyun, S. M., \& Chang, M. (2003). Xanthophylls in microalgae: from biosynthesis to biotechnological mass production and application. Journal of microbiology and biotechnology, 13(2), 165-174.

6. Mouget, J. L., Rosa, P., \& Tremblin, G. (2004). Acclimation of Haslea ostrearia to light of different spectral qualities -confirmation of 'chromatic adaptation' in diatoms. J Photochem Photobiol B, 75(1-2), $1-11$.

7. Terasaki, M., Hirose, A., Narayan, B., Baba,Y., Kawagoe, C.,Yasui, H., et al. (2009). Evaluation of recoverable functional lipid components of several brown seaweeds (phaeophyta) from japan with special reference to fucoxanthin and fucosterol contents. Journal of Phycology, 45(4), 974-980.

8. Ma, L., \& Lin, X-M. (2010). Effects of lutein and zeaxanthin on aspects of eye health. J Sci Food Agric, 90(1), 2-12. 
9. Sachindra, N.M., Sato, E., Maeda, H., Hosokawa, M., Niwano, Y., Kohno, M., et al. (2007). Radical scavenging and singlet oxygen quenching activity of marine carotenoid fucoxanthin and its metabolites. J Agric Food Chem, 55, 8516-8522.

10. Miyashita, K. (2009). Function of marine carotenoids. Forum Nutr, 61 136-146.

11. Miyashita, K., Maeda, H., Okada, T., Abe, M., \& Hosokawa, M. (2010). Anti-obesity and anti-diabetic effects of allenic carotenoid, fucoxanthin. Agro Food, 21, 24-27.

12. Miyashita, K., Nishikawa, S., Beppu, F., Tsukui, T., Abe, M., \& Hosokawa M. (2011). Allenic carotenoid, fucoxanthin, as a novel marine nutraceutical from brown seaweed. J Sci Food Agric, 91(7), 1166-1174.

13. Maeda, H., Hosokawa, M., Sashima, T., Funayama, K., \& Miyashita, K (2005). Fucoxanthin from edible seaweed, Undaria pinnatifida, shows antiobesity effect through UCP1 expression in white adipose tissues. Biochem Biophys Res Commun, 332(2), 392-397.

14. Maeda, H., Hosokawa, M., Sashima, T., \& Miyashita, K. (2007). Dietary combination of fucoxanthin and fish oil attenuates the weight gain of white adipose tissue and decrease blood glucose in obese/diabetic KK-Ay mice. J Agric Food Chem, 55(19), 7701-7706.

15. Maeda, H., Hosokawa, M., Sashima, T., Murakami-Funayama, K., \& Miyashita K (2009). Anti-obesity and anti-diabetic effects of fucoxanthin on diet-induced obesity conditions in a murine model Mol Med Rep, 2(6), 897-902.

16. Hosokawa, M., Miyashita, T., Nishikawa, S., Emi, S., Tsukui, T., Beppu, F., et al. (2010). Fucoxanthin regulates adipocytokine mRNA expression in white adipose tissue of diabetic/obese KK-Ay mice. Arch Biochem Biophys, 504(1), 17-25.

17. Airanthi, M. K., Sasaki, N., Iwasaki, S., Baba, N., Abe, M., Hosokawa, M., et al. (2011). Effect of brown seaweed lipids on fatty acid composition and lipid hydroperoxide levels of mouse liver. J Agric Food Chem, 59(8), 4156-4163.

18. Abidov, M., Ramazanov, Z., Seifulla, R., \& Grachev, S. (2010). The effects of Xanthigen in the weight management of obese premenopausal women with non-alcoholic fatty liver disease and normal liver fat. Diabetes Obes Metab, 12(1),72-81.

19. Onakpoya, I. J., Wider, B., Pittler, M. H., \& Ernst, E. (2011). Food supplements for body weight reduction: a systematic review of systematic reviews. Obesity (Silver Spring), 19(2), 239-244.

20. Pittler, M. H., \& Ernst, E. (2004). Dietary supplements for body-weight reduction: a systematic Review. Am J Clin Nutr, 79(4), 529-536.

21. Asai, A., Sugawara, T., Ono, H., \& Nagao, A. (2004). Biotransformation of fucoxanthinol into amarouciaxanthin A in mice and HepG2 cells: formation and cytotoxicity of fucoxanthin metabolites. Drug Metab Dispos, 32(2), 205-211.

22. Asai, A., Yonekura, L., \& Nagao, A. (2008). Low bioavailability of dietary epoxyxanthophylls in humans. BrJ Nutr, 100(2), 273-277.

23. Tsukui, T., Baba, B., Hosokawa, M., Sashima, T., \& Miyashita, K. (2009). Enhancement of hepatic docosahexaenoic acid and arachidonic acid contents in C57BL/6J mice by dietary fucoxanthin. Fisheries Science, 75(1), 261-263.
24. Matsumoto, M., Hosokawa, M., Matsukawa, N., Hagio, M., Shinoki, A., Nishimukai, M., et al. (2010). Suppressive effects of the marine carotenoids, fucoxanthin and fucoxanthinol on triglyceride absorption in lymph duct-cannulated rats. Eur J Nutr, 49(4), 243-249.

25. Argyropoulos, G., \& Harper, M-L. (2002). Invited review: uncoupling proteins and thermoregulation. Journal of Applied Physiology, 92(5), 2187-2198.

26. Del Mar Gonzalez-Barroso, M., Ricquier, D., \& Cassard-Doulcier, A. M. (2000). The human uncoupling protein-1 gene (UCP1): present status and perspectives in obesity research. Obes Rev, 1(2), 61-72.

27. Mozo, J., Emre, Y., Bouillaud, F., Ricquier, D., \& Criscuolo, F. (2005). Thermoregulation: what role for UCPs in mammals and birds? Biosci Rep, 25(3-4), 227-249.

28. Nedergaard, J., Golozoubova, V., Matthias, A., Asadi, A., Jacobsson, A., \& Cannon, B. (2001). UCP1: the only protein able to mediate adaptive non-shivering thermogenesis and metabolic inefficiency. Biochim Biophys Acta, 1504(1), 82-106.

29. Silva, J. E., \& Rabelo, R. (1997). Regulation of the uncoupling protein gene expression. Eur J Endocrinol, 136(3), 251-264.

30.Cederberg, A., Grønning, L.M., Ahrén, B., Taskén, K., Carlsson, P., \& Enerbäck, S. (2001). FOXC2 is a winged helix gene that counteracts obesity, hypertriglyceridemia, and diet-induced insulin resistance. Cell, 106(5), 563-573.

31. Himms-Hagen, J., Melnyk, A., Zingaretti, M. C., Ceresi, E., Barbatelli, G., \& Cinti, S. (2000). Multilocular fat cells in WAT of CL-316243-treated rats derive directly from white adipocytes. Am J Physiol Cell Physiol, 279(30), C670-C681.

32. Herber, D. (2003). Herbal preparations for obesity: are they useful? Primary Care, 30(2), 441-463.

33. Lenz, T.L., \& Hamilton, W.R. (2004). Supplemental products used for weight loss. J Am Phatm Assoc, 44, 59-67.

34. Jimenez, M., Leger, B., Canola, K., Lehr, L., Arboit, P., Seydoux, J., et al. (2002). Beta(1)/beta(2)/beta(3)-adrenoceptor knockout mice are obese and cold-sensitive but have normal lipolytic responses to fasting. FEBS Lett, 530(1-3), 37-40

35. Kawada, T., Watanabe, T., Takaishi, T., Tanaka, T., \& Iwai, K. (1986). Capsaicin-induced beta-adrenergic action on energy metabolism in rats: influence of capsaicin on oxygen consumption, the respiratory quotient, and substrate utilization. Proc Soc Exp Biol Med, 183(2), $250-256$

36. Watanabe, T., Kawada,T., Yamamoto, M., \& Iwai, K. (1987). Capsaicin, a pungent principle of hot red pepper, evokes catecholamine secretion from the adrenal medulla of anesthetized rats. Biochem Biophys Res Сотmu, 142(1), 259-264.

37. Maeda, H., Hosokawa, M., Sashima, T., Murakami-Funayama, K. \& Miyashita K (2009). Anti-obesity and anti-diabetic effects of fucoxanthin on diet-induced obesity conditions in a murine model. Mol Med Rep, 2(6), 897-902.

38. Lowell, B. B., \& Spiegelman, B. M. (2000). Towards a molecular understanding of adaptive thermogenesis. Nature, 404(6778), 652660. 